\title{
Quelques réflexions sur les relations de Claude Simon à Jean-Paul Sartre
}

Didier Alexandre

\section{(2) OpenEdition \\ 12 Journals}

Édition électronique

URL : http://journals.openedition.org/ccs/571

DOI : $10.4000 /$ ccs.571

ISSN : 2558-782X

Éditeur :

Presses universitaires de Rennes, Association des lecteurs de Claude Simon

\section{Édition imprimée}

Date de publication : 30 avril 2007

Pagination : 87-104

ISBN : 9782354120122

ISSN : 1774-9425

\section{Référence électronique}

Didier Alexandre, "Quelques réflexions sur les relations de Claude Simon à Jean-Paul Sartre », Cahiers Claude Simon [En ligne], 3 | 2007, mis en ligne le 20 septembre 2017, consulté le 07 mai 2019. URL: http://journals.openedition.org/ccs/571; DOI : 10.4000/ccs.571 


\section{Quelques réflexions sur les relations de Claude Simon à Jean-Paul Sartre}

\section{Didier ALEXANDRE*}

La rareté des jugements publiés de Claude Simon sur Jean-Paul Sartre jusqu'aux années soixante est évidente. Cette rareté a pour corollaire la faible intervention de Claude Simon dans le champ littéraire jusqu'aux années soixante : c'est seulement de son entrée aux éditions de Minuit et donc de la publication du Vent que semblent dater les premiers entretiens et les premiers textes critiques de Simon, par exemple pour répondre à l'enquête des Lettres françaises «Qu'est-ce que l'avant-garde? » en novembre $1958^{1}$ - où il n'est nullement question de Jean-Paul Sartre, ni de positionner le Nouveau Roman par rapport à cette notion d'avant-garde vis-à-vis de laquelle Simon se montre prudent, sinon réticent. Le déséquilibre est donc flagrant entre la présence envahissante de Sartre en philosophie, en politique, en littérature et la quasi-absence de Simon. Quand Sartre semble tout ignorer de Claude Simon - il n'existe pas, à ma connaissance, de jugement de Sartre sur les romans de Claude Simon ou sur Claude Simon -, Simon doit considérer Sartre et son œuvre pour définir sa position dans le champ littéraire, comme l'a 
montré la communication de Jean-François Louette. Le premier constat est donc celui-ci : c'est Claude Simon qui ressent le besoin de définir sa pratique d'écrivain par rapport aux interventions publiques de Sartre. Nous pouvons recenser ainsi trois situations où Claude Simon, en 1960, puis 1964, tient à se différencier de l'écrivain-philosophe. Il faut ensuite tenter de comprendre les raisons qui dictent ce positionnement, ce qui n'est pas facile.

La première manifestation conflictuelle de Claude Simon à l'égard de Jean-Paul Sartre intervient dans l'entretien accordé à Madeleine Chapsal le 10 novembre 1960 pour L'Express, un hebdomadaire qui défend des valeurs de gauche et des valeurs littéraires nouvelles. On trouve dans cet entretien, comme dans le texte rédigé des Lettresfrançaises de 1958, l'admiration vouée à Michel Leiris, Fourbis, et à Alain Robbe-Grillet, « la seule tentative d'une littérature purement matérialiste », un " effort prodigieux pour se débarrasser des miasmes, des mythes qui nous encombrent» ${ }^{1}$. Claude Simon souscrit ainsi au projet de Robbe-Grillet d'un roman œuvre d'art autosuffisante et donc purifiée : c'est bien ce qu'évoquent les miasmes de toute signification extérieure à elle. Ce principe d'autosuffisance est affirmé dès 1956 dans "Sur quelques notions périmées » (1957), puis dans « Nature, humanisme et tragédie» $(1958)^{2}$. Il trouve sa formulation dans le rejet du roman fondé sur la philosophie, de Camus ou de Sartre : Claude Simon cite en 1958 dans Les Lettresfrançaises le jugement formulé par Robbe-Grillet dans «Une voie pour le roman futur» de 1957 : «Le monde n'est ni signifiant ni absurde. Il est, tout simplement. [...] Autour de nous, défiant la meute de nos adjectifs

\footnotetext{
1 Madeleine Chapsal, «Entretien avec Claude Simon », L'Express, 10 novembre 1960.

${ }^{2}$ Voir Alain Robbe-Grillet, Pour un nouveau roman, Paris, Minuit, 1963 : « L'existence d'une œuvre d'art, son poids, ne sont pas à la merci de grilles d'interprétation qui coïncideraient, ou non, avec ses contours. L'œuvre d'art, comme le monde, est une forme vivante : elle est, elle n'a pas besoin de justification. Le zèbre est réel, le nier ne serait pas raisonnable, bien que ses rayures soient sans doute dépourvues de sens. Il en va de même pour une symphonie, une peinture, un roman : c'est dans leur forme que réside leur réalité. » (p. 41) ; «Le crime, c'est d'affirmer qu'il existe quelque chose, dans le monde, qui n'est pas l'homme, qui ne lui adresse aucun signe, qui n'a rien de commun avec lui. » (p. 47), etc.
} 
animistes, ou ménagers, les choses sont là. $\|^{1}$ - Claude Simon réserve, de la même manière, les italiques ou les majuscules au verbe être : "L'artiste de 1958 réalise chaque jour un peu plus que le monde "EST ABSOLUMENT" $»^{2}$. Simon, sur les traces de Robbe-Grillet, écarte donc toute intentionnalité de sens et toute interprétation philosophique du monde : il ne retient de la phénoménologie que l'êtrelà du monde et la relation d'altérité que cela implique.

C'est à cette même démarche d'accord avec Robbe-Grillet et de prise de distance vis-à-vis de Sartre que, dans "Le jeune roman » de Madeleine Chapsal, publié dans L'Express du 12 janvier 1961, Claude Simon revient. Très direct, il insiste sur l'accord « sur tous les points » qui règne entre lui et Robbe-Grillet. Et il construit son argumentation contre Jean-Paul Sartre. En effet, Simon a donné le 11 janvier 1961, en Sorbonne, « une conférence intitulée Signification, roman et chronologie », dont on peut noter, dès le titre, les échos sartriens. Car la conférence de Simon dialogue avec les essais de Sartre : «Vous ne pouvez pas savoir le mal que ça m'a donné de réfléchir à ces choses, moi qui ne m'intéresse pas aux idées, n'en ai jamais une en tête... ». Madeleine Chapsal ajoute qu'«il a relu Matérialisme et révolution de Jean-Paul Sartre (1946), Qu'est-ce que la littérature ? (1947), et d'autres textes du même », citant de nouveau Simon : «J'ai toute la collection des Temps modernes à la maison, depuis le premier numéro. $»^{3}$. Les reproches adressés à Sartre portent sur la signification, « une interprétation des choses qui tendrait à prouver que le monde a un sens » et donc sur l'idée sartrienne que le roman présuppose une métaphysique. L'argumentaire de Simon reprend celui de Robbe-Grillet, puisqu'il congédie les croyants et les marxistes, et encore les romantiques ${ }^{4}$, comme cela est fait dans Pour un nouveau roman. La conférence donnée à la Sorbonne annexe même, au nombre des croyants, Jean Genet (Notre-Dame desfleurs). Elle condamne, comme l'entretien de L'Express, la finalité utilitaire et morale de la littérature : «Tous les mots d'ordre de la littérature engagée [...] posent

\footnotetext{
1 Ibid., p. 18.

2 Les Lettres françaises, $\mathrm{n}^{\circ} 717,24-30$ avril 1958.

3 Madeleine Chapsal, «Le jeune roman », L'Express, 12 janvier 1961, p. 31.

4 Ibid., p. 32.
} 
le problème en termes de morale et considèrent qu'un écrivain doit répondre à une certaine demande. ». Simon définit ainsi en creux, contre la position sartrienne, sa propre écriture intransitive : « La totale inefficacité dans le domaine de l'immédiat lui semble une nécessité pour l'écrivain, c'est l'art pour l'art sans réserve - un art qui se doit de traduire et de ne traduire que "des réactions sensorielles" " ${ }^{1}$. Tandis que l'utilité condamne tout roman, de Vercors ou de Richard Wright, à n'être rapidement plus lu, le roman d'art continue de toucher les lecteurs des autres générations : au critère de l'utilité, Simon oppose celui du jugement de goût ${ }^{2}$. Il dit, du reste, trouver dans les déclarations de Sartre à Claude Sarraute, faites dans $L e$ Monde au moment de la mise en scène des Séquestrés d'Altona, et dans la Préface écrite en 1960 à Aden-Arabie de Paul Nizan, son constat de l'impuissance de la littérature qui " aboutit finalement à l'échec", à ce « néant où nous a fait émerger la littérature du $\mathrm{XIX}^{\mathrm{e}}$ siècle $»^{3}$. Le dialogue n'est donc pas stérile : il reconstruit une histoire littéraire qui fait de Sartre l'héritier d'un romantisme transitif, messianique, ouvert sur l'histoire, dont la Seconde Guerre mondiale marque la fin catastrophique, un héritier qui ne peut se satisfaire de l'esthétisme littéraire du Nouveau Roman en demeurant conscient de l'échec de la littérature.

Deux types de textes de Sartre sont convoqués : le premier est évidemment Qu'est-ce que la littérature ? publié dans Les Temps modernes de février à juillet 1947 et repris dans Situations II en 1948 ; le second type de texte consiste en des écrits plus ponctuels, de circonstance, par exemple la préface écrite pour la réédition d'Aden-Arabie de Paul Nizan, qui s'inscrit parmi un ensemble simultané d'oraisons funèbres, à Camus, à Merleau-Ponty, à Frantz Fanon.

Je ferai quelques commentaires sut Qu'est-ce que la littérature?. Il est vrai que dans Qu'est-ce qu'écrire ? Sartre, en opposant prose et poésie, définit la prose «utilitaire par essence», le prosateur « comme

1 Janine Parot, « À la Sorbonne, Claude Simon part en guerre contre la signification », Les Lettres françaises, $\mathrm{n}^{\circ}$ 859,19-25 janvier 1961.

${ }^{2}$ Madeleine Chapsal, « Le jeune roman », art. cit., p. 32, et Janine Parot, art. cit.

3 Janine Parot, art. cit. 
un homme qui se sert des mots", sa matière comme " naturellement signifiante », et exclut le critère de goût, puisqu'« il ne s'agit pas de savoir si [les mots] plaisent ou déplaisent en eux-mêmes ", au profit d'une fonction, très husserlienne, d'indication, à la fois didactique et déïctique : "[il s'agit de savoir si les mots] indiquent correctement une certaine chose du monde ou une certaine notion. $»^{1}$. C'est bien ce texte que Claude Simon cite dans le Discours de Stockholm, pour définir son roman en s'opposant à Sartre qui demeure vingt ans après une référence incontournable - ce qui, dans l'enceinte même du Nobel, est pour le moins singulier. Le gauchissement de la pensée de Sartre est manifeste, puisque la théorie de la prose est déplacée sur le roman - on passe d'une forme à un genre - et que la nature même du sens que doit véhiculer l'œuvre en prose selon Sartre est comprise comme un savoir et non plus comme la parole d'un homme en situation dans le monde et donc comme une praxis au sens sartrien ${ }^{2}$. On peut ainsi comparer ces deux citations

N'a-t-on pas coutume de poser à tous les jeunes gens qui se proposent d'écrire cette question de principe : «Avez-vous quelque chose à dire ? » Par quoi il faut entendre : quelque chose qui vaille la peine d'être communiqué. Mais comment comprendre ce qui en "vaut la peine» si ce n'est par un recours à un système de valeurs transcendant ?

L'œuvre en prose est donc transitive, et elle n'est pas dénuée de style : au contraire, selon Sartre, le prosateur cherche les moyens de communiquer, ce qui implique pour le moins une rhétorique (redoutable chez Sartre). Simon donne un sens restrictif au verbe avoir, lui ôtant tout sens de nécessité ou d'obligation morale, inséparable de la responsabilité :

Dépositaire ou détenteur privilégié par l'effet de cette grâce d'un savoir ("Qu'avez-vous à dire ?» demandait Sartre - en d'autres termes : «Quel savoirpossédez-vous?»), dépositaire

1 Situations II, Qu'est-ce que la littérature?, Paris, Gallimard, 1948, coll. "Idées », 1976, p. 26. On ne saurait confondre utilité et utilitarisme bourgeois chez Sartre (p. 147).

2 Ibid., p. 286-287.

3 Ibid., p. 28. 
donc avant même d'écrire d'une connaissance refusée au commun des mortels, l'écrivain se voit assigner la mission de les en instruire, et le roman va tout naturellement prendre la forme imagée sous laquelle est délivré l'enseignement religieux, celle de la parabole, de la fable. (DS, 891-2)

De même, en construisant une histoire du roman qui met en perspective le roman du XIX ${ }^{\mathrm{e}}$ siècle, Malraux et Sartre, Simon oppose un réalisme qui perdurerait jusqu'au second conflit mondial et s'épuiserait dans la prose selon Sartre à un roman qui suit « d'autres voies ». Dans "les bibliothèques de gares ou ailleurs", où " on continue, et on continuera encore longtemps, à vendre et à acheter par milliers d'aimables ou de terrifiants récits d'aventures à conclusions optimistes ou désespérées, et aux titres annonceurs de vérités révélées comme par exemple La Condition humaine, L'Espoir ou Les Chemins de la liberté... » (DS, 892), on peut évidemment ranger les romans évoqués dans la séquence de la lecture que fait une voyageuse dans le train de La Bataille de Pharsale ${ }^{1}$. Or, si Jean-Paul Sartre, dans le texte de Présentation des Temps modernes, rejette «l'art pour l'art» auquel, précisément, se réfère Simon, nous l'avons vu, il rejette aussi le réalisme et le déterminisme qui le sous-tend, ce qu'il reprend dans Qu'est-ce que la littérature? où Flaubert est présenté comme un styliste «amant pur de la forme et père du naturalisme». Tout se passe comme si Claude Simon accablait Sartre de ce dont Sartre veut constamment se différencier dans Qu'est-ce que la littérature? par une approche historique et sociologique de la littérature.

Le second type de textes cités par Claude Simon est la conséquence même de la fonction donnée à la littérature par Sartre. Dans l'entretien avec Madeleine Chapsal de L'Express du 12 janvier 1960, Simon rappelle que Sartre a déclaré à Claude Sarraute pour Le Monde renoncer à l'écriture des romans au moment de la mise en scène des Séquestrés d'Altona, et à Madeleine Chapsal elle-même l'expérience de totalité que doit être la littérature : «Si la littérature n'est pas tout, elle ne vaut pas une heure de peine. C'est cela que je veux dire par enga-

${ }^{1}$ La Bataille de Pharsale, p. 675 et p. 678. Sartre rejette la littérature de consommation au profit d'une littérature de production qu'il qualifie de "littérature totale» (Qu'est-ce que la littérature?, op. cit., p. 288-289) 
gement. Elle sèche sur pieds si vous la réduisez à l'innocence, à des chansons. Si chaque phrase écrite ne résonne pas à tous les niveaux de l'homme et de la société, elle ne signifie rien. La littérature d'une époque, c'est l'époque digérée par la littérature, $»^{1}$. Pensée comme praxis, c'est-à-dire comme "action dans l'histoire et sur l'histoire", comme volonté de "faire l'histoire ", " comme synthèse de la relativité historique et de l'absolu moral et métaphysique, avec ce monde hostile et amical, terrible et dérisoire qu'elle nous révèle $»^{2}$, ce que l'on pourrait opposer à l'épigraphe que choisit Claude Simon pour L'Herbe, l'écriture sartrienne est soumise au devenir historique. Ancrée dans l'après-guerre, l'année 1947, la première définition donnée est soumise à l'héritage dramatique de la Seconde Guerre mondiale : la référence à Auschwitz est aussi celle de Sartre $^{3}$. La notion de Mal n'est pas tant morale, chez Sartre, qu'historique, et doit être comprise comme ce qui est oppression faite à l'humain libre : « Mais d'autre part, battus, brûlés, aveuglés, rompus, la plupart des résistants n'ont pas parlé ; ils ont brisé le cercle du Mal et réaffirmé l'humain, pour eux, pour nous, pour leurs tortionnaires mêmes. Ils l'ont fait sans témoins, sans recours, sans espoir, souvent même sans foi. $»^{4}$. Se fait donc jour, chez Sartre, la tentation du récit témoignage dans le présent historique, qui appelle l'invention de nouvelles formes d'écriture narrative, et que réalisera la revue des Temps modernes en insérant des récits de vie, en particulier des témoignages de guerre. On peut du reste se demander si Claude Simon, lorsqu'il écrit $L a$ Corde raide, n'opte pas pour cette forme de récit témoignage, à cette différence près que l'individu écrivain de Simon ne s'affirme pas comme «action constructrice, créatrice " niant l'aliénation de l'homme par les mouvements de l'histoire et faisant l'histoire ${ }^{5}$. Cette dialectique est absente de la posture simonienne. À travers la référence à Cézanne et au corps de Cézanne, sa main et son œil, l'art est présenté en rupture avec l'histoire présente et comme fin en soi. En conséquence,

1 Madeleine Chapsal, Les écrivains en personne, Julliard, 1960, p. 211. Sur cette littérature totale, synthèse du faire, de l'avoir, de l'être, voir Qu'est-ce que la littérature?, op. cit., p. 289.

${ }^{2}$ Qu'est-ce que la littérature?, op. cit., p. 287-288.

3 Ibid., p. 262.

4 Ibid., p. 265.

5 Ibid., p. 283. 
et ce serait évidemment un voie à explorer, le dialogue Sartre-Simon commencerait dès les années immédiatement postérieures à la fin de la Seconde Guerre mondiale. Chez Sartre, l'éthique humaine l'humanisme tragique que dénonce chez Sartre Alain Robbe-Grillet dans Pour un nouveau roman ${ }^{1}$ - précède l'écriture : le prototype de l'oppression l'emporte. L'acte d'écrire est constamment inséparable de la défense des opprimés, en 1947 (relation bourreau-victime) ou dans les années soixante, et son efficacité dans l'histoire en est la meilleure mesure - et donc la pire. Le renoncement au roman de Sartre correspond autant à l'épuisement du genre romanesque qu'à une réflexion lucide sur la possibilité d'un art engagé, c'est-à-dire confronté aux réalités de l'histoire sociale et économique, à l'échelle locale et mondiale. Sartre n'est plus alors un écrivain : il met au service d'un combat contre l'oppression le capital acquis par la littérature, ce qu'a parfaitement vu Maurice Blanchot ${ }^{2}$.

La question de l'engagement est évidemment au centre du débat, surtout en ces années soixante où Sartre et Simon sont parmi les signataires du manifeste des $121^{3}$. Dans l'entretien avec Madeleine Chapsal publié dans L'Express du 10 novembre 1960, Claude Simon commente sa signature, en réponse à cette question : "Vous repoussez les idées, mais n'êtes-vous pas signataire d'un certain manifeste? ».

Avant d'examiner la réponse de Simon, il faut rappeler que le procès Jeanson concernait un collaborateur des Temps modernes, commentateur de Sartre, auteur en 1955 d'Algérie hors la loi, qui, brouillé avec Sartre après la condamnation par le philosophe de l'intervention soviétique en Hongrie en 1956, fait cependant appel à

1 Pour un nouveau roman, op. cit., p. 56 sv.

2 «Les intellectuels en question», Le Débat, novembre 1984, p. 27.

${ }^{3}$ C'est le 4 septembre 1960 que Le Monde annonce que « cent vingt et un écrivains et artistes ont signé une déclaration sur le "droit à l'insoumission dans la guerre d'Algérie" ». Maurice Blanchot, Jean Schuster, Dionys Mascolo, Maurice Nadeau, Jean Pouillon sont à l'origine du projet. Mascolo et Schuster, puis Blanchot le rédigèrent. Or, c'est le 6 septembre 1960 que s'ouvrait le procès Jeanson, c'est-àdire des réseaux de soutien au F.L.N. Le manifeste réunissait des signataires venus d'horizons très divers : milieux surréalistes, Temps modernes, Lettres nouvelles, anciens communistes, Minuit. 
lui en 1959 pour obtenir son soutien en faveur du peuple algérien. JeanPaul Sartre est absent de France lors du procès : il est au Brésil où il ne manque pas de plaider pour l'anti-colonialisme et pour le combat du F.L.N., et envoie le 16 septembre 1960 un télégramme pour excuser son absence, et dire sa solidarité avec les accusés, tandis que Roland Dumas lit une lettre de lui de soutien aux accusés, qui provoque un immense tollé : «Une bombe », titre L'Aurore ${ }^{1}$. Sartre se trouve donc au cœur du procès, du manifeste et des troubles qui s'ensuivirent, tel le saccage des vitrines de L'Express le 3 octobre 1960. On sait aussi que les éditions de Minuit, qui avaient publié La Question de Jean Alleg, furent très sérieusement menacées. L'attribution du Prix de l'Express à Claude Simon pour La Route des Flandres en 1961 doit être replacée dans ce contexte : elle signifie aussi le lien établi entre le manifeste, le procès Jeanson, l'hebdomadaire, la maison d'édition, à travers une œuvre qui fait une place discrète, mais réelle aux combattants venus du Nord de l'Afrique, rappelant et la part qu'ils ont prise en 1940 dans la lutte contre l'armée allemande et l'hostilité raciste dont ils sont l'objet de la part de certains combattants français ${ }^{2}$. L'écriture allusive de Simon, en fait indirecte dans la mesure où le procès mené contre l'institution militaire dans La Route des Flandres peut renvoyer à l'actualité algérienne, diverge donc de celle de Sartre qui multiplie les textes qui apostrophent les Français sur la question du colonialisme et du tiers monde, par exemple, la Préface aux Damnés de la terre de Frantz Fanon ${ }^{3}$, immédiatement contemporaine de La Route des Flandres.

1 Sur ce point, voir Annie Cohen-Solal, Sartre, 1905-1980, Gallimard, 1985, p. 537547.

2 Cf. le maquereau d'Alger, juif, « le roi venu tout droit de la Bible » qui défie le pouvoir allemand dans le camp le jour du Yom Kippour (RF, 344-5).

3 Les Cahiers libres, $\mathrm{n}^{\circ}$ 27-28, Maspero, 1961. Voici deux citations : « Nos chères valeurs perdent leurs ailes ; à les regarder de près, on n'en trouvera pas une qui ne soit tachée de sang. S'il vous faut un exemple, rappelez-vous ces grands mots Que c'est généreux la France! Généreux, nous ? Et Sétif? Et ces huit années de guerre féroce qui ont coûté la vie à plus d'un million d'Algériens ? Et la gégène ?» (Situations V, Paris, Gallimard, 1965, p. 188) ; «Les marques de la violence, nul ne les effacera : c'est la violence seule qui peut les détruire. Et le colonisé se guérit de la névrose coloniale en chassant le colon par les armes » \{ibid., p. 167). 
La réponse que Simon fait à Madeleine Chapsal vise à réconcilier l'écriture simonienne et la signature du manifeste des 121. L'auteur de La Route des Flandres attribue dans un premier temps son geste à «des mouvements du cœur»; «Je n'ai jamais pu supporter l'injustice, qu'on batte quelqu'un, qu'on humilie ou que l'on fasse souffrir... J'ai signé ce manifeste, je suis inculpé, je continuerai.». C'est ce pouvoir d'indignation qui, lors du procès Jeanson, a dicté son témoignage :

L'autre jour, au procès Jeanson où je témoignais, le président du tribunal m'a menacé d'expulsion parce que, paraît-il, j'insultais l'Armée française en disant que nous poursuivions en Algérie une guerre injuste avec des moyens indignes d'une nation civilisée. C'est suffocant. J'ai été tenté de lui répondre qu'en somme, il avait raison d'interdire que l'on appelât cela une "guerre". Parce que cinq cent mille hommes pourvus d'avions, de canons, de tanks, contre cinq mille malheureux armés de fusils de chasse ou même de fusils mitrailleurs, effectivement, ce n'est pas de la guerre mais de l'assassinat. ${ }^{1}$

À quoi fait suite une critique de l'armée et des officiers français. Mais dans un second temps, il avance la thèse suivante :

L'incessante remise en question, l'incessante contestation de ce qui est tenu pour acquis. Signer la Déclaration des 121, c'était contester la légitimité de certains actes : tuer dans une guerre injuste, torturer. De la même façon, écrire (bien entendu dans la mesure où l'on parvient à faire œuvre d'art) c'est contester les formes et les rapports établis, reconnus, consacrés... ${ }^{2}$

Claude Simon s'écarte donc de la signification que Sartre donne à l'engagement par l'écriture :

Je dirai qu'un écrivain est engagé lorsqu'il tâche à prendre la conscience la plus lucide et la plus entière d'être embarqué, c'est-à-dire lorsqu'il fait passer pour lui et pour les autres

1 Madeleine Chapsal, «Entretien avec Claude Simon », L'Express, 10 novembre 1960.

2 Ibid. 
l'engagement de la spontanéité immédiate au réfléchi. L'écrivain est médiateur par excellence et son engagement est la médiation. Seulement s'il est vrai qu'il faut demander des comptes à son œuvre à partir de sa condition, il faut se rappeler aussi que sa condition n'est pas seulement celle d'un homme en général mais précisément aussi d'un écrivain. [...] nul n'est obligé de se choisir écrivain. ${ }^{1}$

Lorsque ce choix est fait, l'écrivain, qui a une fonction sociale, est donc tenu de répondre à une attente et une demande des hommes. Dans une approche sociocritique, Sartre noue ensemble l'écrivain et l'homme, tandis que Simon les dissocie. L'homme, fort éventuellement du capital acquis par l'écrivain, peut intervenir et s'engager dans des entretiens qui défient la censure: mais l'écrivain s'engage dans un autre espace, discursif, supposant une anthropologie où l'homme est constitué des langages multiples qu'il tient. Les références faites par Claude Simon à Roland Barthes ne sont donc pas insignifiantes : elles font sens en fonction de cette sémiologie et de cette conception structuraliste de l'humain, telle que l'écriture est définie en termes de révolution. La subversion des discours qui prétendent à la légitimité - et on notera aussi combien le terme anticipe la pensée sur les discours de Lyotard - est révolutionnaire et tend à redéfinir l'humain. Sur ce point, Simon est proche de l'engagement tel que le définit Alain Robbe-Grillet qui renvoie dos à dos l'engagement en faveur de valeurs religieuses et l'engagement en faveur de valeurs sociales, Mauriac et Jdanov, et qui mesure les limites de l'engagement sartrien fondé sur la double liberté de l'écrivain et du lecteur:

Redonnons donc à la notion d'engagement le seul sens qu'elle peut avoir pour nous. Au lieu d'être de nature politique, l'engagement c'est, pour l'écrivain, la pleine conscience des problèmes actuels de son propre langage, la conviction de leur extrême importance, la volonté de les résoudre de l'intérieur. C'est là, pour lui, la seule chance de demeurer un 
artiste, et, sans doute aussi, par voie de conséquence obscure et lointaine, de servir un jour peut-être à quelque chose peut-être même à la révolution. ${ }^{1}$

À la révolution dans l'histoire, qui demeure l'horizon lointain de Sartre, s'oppose la révolution du langage, qui deviendra un des arguments de Tel quel. C'est bien cette thèse que développe Claude Simon lors du Congrès de Vienne d'avril 1967 qui avait pour sujet "Littérature : tradition et révolution", dans un texte publié dans Ta Quinzaine littéraire (1-15 mai 1967). L'ombre de Sartre demeure présente derrière les formulations de Simon :

Il convient de mettre fin à une légende : jamais aucune œuvre d'art, aucune œuvre littéraire n'a eu, dans l'immédiat., un poids quelconque sur le cours de l'Histoire. [...] En revanche, au sein de l'immense et incessante gestation du monde, et dans l'ensemble des activités de l'esprit, toute production de celuici, à condition d'apporter quelque chose de neuf, joue son rôle, le plus souvent de façon invisible, souterraine, mais cependant capitale.

On peut, en conséquence, relire Histoire, contemporain de ce texte, comme la dérision d'une écriture engagée, en l'occurrence celle de John Reed, Dixjours qui ébranlèrent le monde, qui conduit le narrateur, jeune, à Barcelone, ou qui s'épuise dans la figure de compromission qu'est le personnage de Lambert, et comme une révolution en acte des langages. Le dialogue avec Sartre, en ces années, ne cesse donc pas : si les deux écrivains se rencontrent sur la fin des voyages et du tourisme, leur approche du colonialisme et du tiers-monde diverge radicalement, Sartre la faisant en termes économiques, politiques, culturels (valeurs, arts), Claude Simon la faisant à travers les symboles que sont les cartes postales et les textes dont elles sont le support. L'Aden d'Histoire est par nature différent de l'Aden d'Aden-Arabie. De même, dans Les Corps conducteurs, le récit de la conférence d'écrivains à laquelle assiste le personnage reprend, de manière dérisoire, les débats sur la littérature et la

Pour un nouveau roman, "Sur quelques notions périmées », p. 39. Voir aussi le texte de Alain Robbe-Grillet le voyageur, Christian Bourgois éditeur, 2001, "Littérature et politique » (1963), p. $65 \mathrm{sv}$ (publié dans L'Express). 
révolution, la nationalité littéraire, sur fond de colonialisme et de luttes issues du colonialisme : l'épuisement des intervenants résume assez bien le jugement de Simon sur ces questions, comme la nausée du personnage, clin d'œil possible à Sartre. Enfin, le récit L'Invitation doit être rangé dans la série des nombreux retours d'U.R.S.S., ceux de Barbusse, Aragon, Malraux, Bloch et Sartre, qui dans Libération, du 15 au 20 juillet 1954, livre les impressions euphoriques faites par son séjour en U.R.S.S.

La posture simonienne consiste en une écriture sur l'histoire à distance de l'histoire. Et ceci est bien inscrit dans l'œuvre. Dans Le Jardin des Liantes, Claude Simon représente S. présent, à distance des fêtes organisées par Leiris. Dans la mise en scène le 16 juin 1944 de la pièce de Picasso, Le désir attrapé par la queue, mise en scène réalisée par Albert Camus, Sartre joue le rôle du «Bout rond » et Simone de Beauvoir de «La Cousine $»^{1}$. Simon est donc en marge, dans une spatialité référentielle et symbolique, des milieux surréalistes - ce qui apparaît ailleurs dans l'œuvre, que ce soit dans Gulliver où le personnage de Max a un moment fréquenté ces milieux parisiens avant de céder à son homosexualité qui le mène au suicide - faut-il voir en Max une allusion à Cocteau, qui sera mentionné dans Le Jardin des Liantes, ou un retour sur la question de l'homosexualité et de l'art posée par Sartre dans le Saint Genet comédien et martyr publié en 1952, l'année de publication de Gulliver $^{2}$-, ou que ce soit dans Histoire, à travers l'exemple de l'oncle Charles, dont l'aventure parisienne en

1 Sur la question, voir Annie Cohen-Solal, Sartre, 1905-1980, op. cit., p. 278-280. Le jardin des Plantes, p. 1146-1147, 1152-1154.

2 Gulliver, Paris, Calmann-Lévy, 1952, p. 120-121, p. 258-262. Sartre, dans son Explication de L'Etranger, écrit : "Le récit de M. Camus est analytique et humoristique. [...] Qu'il nous suffise de marquer que l'univers de l'homme absurde est le monde analytique des néo-réalistes. Littérairement le procédé a fait ses preuves : c'est celui de L'Ingénu ou de Micromégas; c'est celui de Gulliver. Car le XVIII ${ }^{e}$ siècle a eu aussi ses étrangers, - en général de "bons sauvages" qui, transportés dans une civilisation inconnue, percevaient les faits avant d'en saisir le sens. L'effet de ce décalage n'était-il pas précisément de provoquer chez le lecteur le sentiment de l'absurde ? M. Camus semble s'en souvenir à plusieurs reprises, en particulier quand il nous montre son héros réfléchissant sur les raisons de son emprisonnement. » (Situations I, Paris, Gallimard, 1947, p. 116-117). Gulliver, c'est le personnage de Max, qu'il faudrait en conséquence situer par rapport à l'étranger, à la question de l'absurde et à la lecture sartrienne de L'Etranger. 
milieux artistiques s'achève de nouveau par un suicide, cette fois celui de l'épouse. Littéralement, la littérature, posée comme absolu, ne conduit qu'à la mort et à la fin de la littérature. Le départ pour la province originaire marque ainsi, dans l'œuvre, le refus des milieux littéraires festifs et mondains et la recherche d'une voie solitaire, austère, laborieuse, sans plaisir réel. Au brillant sartrien et parisien s'oppose le sérieux simonien, provincial.

Ces mises en fiction de l'avant-garde - qui apportent une réponse à l'enquête faite par Les Lettresfrançaises en 1958 - montrent aussi l'extrême méfiance, précoce, de Simon à l'égard des mouvements et des groupements littéraires. Littéralement, l'avant-garde s'épuise dans le spectacle, n'offrant d'issue que dans le suicide, la mort de l'écrivain. Les avant-gardes construisent une histoire littéraire faite de revirements ponctuels, comme en témoigne la relecture faite de Paul Claudel dans les milieux surréalistes selon Claude Simon ${ }^{1}$. Ce qui se dit ainsi, dans les romans eux-mêmes, c'est le passage d'une histoire littéraire fondée sur des mouvements et des critiques d'auteurs - ce qui est parfaitement intégré par Sartre dans Qu'est-ce que la littérature ? - à une pensée du littéraire fondé sur une critique d'auteurs qui annexe le discours d'une critique savante. C'est ce qu'illustre le second débat important qui oppose Sartre et Simon à la fin de l'année 1964. Dans une interview accordée à Jacqueline Piatier et publiée dans Le Monde du 18 avril 1964, Sartre donne sa conception de la littérature en 1964. Il constate : «On n'est pas plus sauvé par la politique que par la littérature. » Son engagement dans le tiers-mondisme impose des constats : «L'univers reste noir. Nous sommes des animaux sinistrés... Mais j'ai découvert brusquement que l'aliénation, l'exploitation de l'homme par l'homme, la sousalimentation, reléguaient au second plan le mal métaphysique, qui est un luxe. La faim, elle, est un mal tout court. ». C'est fort de cette question qu'il se demande s'il est possible de «lire Robbe-Grillet dans un pays sous-développé » et affirme : «En face d'un enfant qui meurt, La Nausée ne fait pas le poids. $»^{2}$. La logique sartrienne

1 Le Jardin des Plantes, p. 1146. Jean-Louis Barrault avait mis en scène Le Soulier de satin à la Comédie française en 1943.

2 «Jean-Paul Sartre s'explique sur Les Mots», Le Monde, 18 avril 1964. 
est donc radicale et sans appel : l'écrivain « doit donc se ranger au côté du plus grand nombre, des deux milliards d'affamés, s'il veut pouvoir s'adresser à tous et être lu par tous. Faute de quoi, il est au service d'une classe privilégiée et exploiteur comme elle...». C'est à cette déclaration, qui fait scandale dans les milieux littéraires, que répondent Yves Berger et Claude Simon dans L'Express du 28 mai 1964. L'argumentation de Claude Simon se fonde sur son vécu (la faim n'empêchait pas les prisonniers dans le camp en 1940 de lire) et son expérience de l'écriture, théorisée par la distinction que fait Barthes dans Le Degré zéro de l'écriture:

L'écrivain est celui dont l'action s'exerce sur son propre instrument, le langage : il travaille sa parole (j'ajouterais qu'il est aussi travaillé par elle), absorbant le pourquoi du monde dans un comment écrire. L'écrivant est celui qui s'approprie le langage de l'écrivain à des fins politiques.

Pour lui la parole n'est qu'un moyen : elle supporte un faire, elle ne le constitue pas. ${ }^{1}$

Simon définit donc son écriture dans un rapport à la langue comme aventure et découverte, que métaphorise la référence au topos littéraire de Colomb, dont on sait quelle importance il prendra dans Orion aveugle. Simon revient ainsi sur la question des significations, "floues, imprécises, vacillantes", pour se demander "comment imaginer que, par le fonctionnement de ce mécanisme structural par lequel se fait l'œuvre de l'écrivain [...] pourraient se dégager des significations utilitaires immédiatement consommables $»^{2}$. Cette argumentation permet donc de répondre aux reproches adressés par Sartre à Robbe-Grillet, qui cherche à « découvrir (dans le sens littéral du mot) un univers tangible, mesurable, lavé, nettoyé des vieux mythes qui chloroforment l'humanité $»^{3}$. Enfin, Simon associe l'échec de la littérature selon Sartre à une morale de dévots et au projet révolutionnaire marxiste, semblant ignorer que Sartre a depuis longtemps pris ses distances par rapport au modèle

1 « Deux écrivains répondent à Jean-Paul Sartre », « Pour qui donc écrit Sartre ? », L'Express, 28 mai 1964, p. 32.

2 Ibid.

3 Ibid. 
soviétique. L'art, selon Simon, demeure nécessaire à l'homme et à la société dont le paradigme demeure étonnamment l'univers concentrationnaire : les " ouvertures sur les espaces toujours libres et inaliénables de l'imaginaire, du merveilleux et du songe » rendent impossible une société "aussi cauchemardesque qu'un immense camp de concentration fonctionnel et bien organisé où la nourriture serait abondante, le travail peu pénible, les couvertures chaudes, les toits des baraques étanches, et où croupiraient non plus des hommes mais des bêtes ». Ce débat laisse des traces dans l'œuvre, puisque Claude Simon revient sur la question du Musée dans Histoire, La Bataille de Pharsale, et Les Corps conducteurs. Fidèle à sa définition de la révolution du langage opérée à l'intérieur de la tradition, il ajoute cet autre élément, la nécessité de l'art à l'homme comme élément qui le différencie de l'animalité. C'est donc bien une anthropologie, où la fonction symbolique constitue l'humanité et son devenir, que construit Simon.

On sait que cette polémique, dont on ne doit pas minimiser la virulence, eut pour épilogue, le 9 décembre 1964 à la Mutualité, un débat organisé autour de la question «Que peut la littérature ? »par le journal Clarté, organe de l'Union des étudiants communistes ${ }^{2}$. Claude Simon, invité, déclina l'invitation ${ }^{3}$. Sartre, dont l'autobiographie Les Mots était un succès littéraire incontestable, était alors auréolé et stigmatisé pour le refus du Prix Nobel qui lui avait été attribué en octobre 1964. Le débat mériterait à lui seul une étude, car il marque une réelle fracture dans le champ littéraire romanesque. On notera simplement que Jean Ricardou reprit l'argumentation développée par Claude Simon dans son article de L'Express. JeanPierre Faye, quant à lui, s'attacha à distinguer l'écrivain du militant, et à donner pour fonction à l'écriture révolutionnaire d'assurer une

\section{Ibid., p. 33.}

2 Et non en 1965 comme l'écrit Francine Dugast-Portes dans Le Nouveau Roman, une césure dans l'histoire du récit, Nathan Université, 2001, p. 153.

3 Sur les circonstances, voir le récit de Claude Simon, "Lettre ouverte à l'Union des étudiants communistes», L'Express, 7-13 décembre 1964, p. 81. Simon dit avoir suggéré des noms d'amis, deux : on peut penser que l'un d'entre eux était Jean Ricardou, alors membre de Tel quel. 
meilleure communication sociale - ce qui, soit dit en passant, ne diverge que bien peu des objectifs sartriens. Aux étudiants de Clarté qui récusaient les thèses de Jdanov et confondaient révolutionnaire et écrivain, dans un combat pour " transformer» la réalité, « là où l'histoire se fait», Semprun répond par la référence à l'autotélisme de Robbe-Grillet et l'intransitivité de l'écriture. Le débat consacre donc le recul d'une conception marxiste de l'écriture romanesque en prise sur l'histoire présente. Au contraire, il témoigne de l'émergence d'un discours critique fondé sur une critique savante et sur une nouvelle anthropologie à laquelle se rallie Claude Simon. Sartre le sait, puisque dans l'année 1966, à la suite de la publication de Les Mots et les choses de Foucault, il revient sur le structuralisme pour rejeter le refus de l'histoire et du marxisme ${ }^{1}$. Deux oppositions fortes se dégagent: la première porte sur la nécessaire dialectique selon Sartre de la structure et de l'histoire; la seconde porte sur l'identification, ou son rejet, de la pensée au langage et donc sur le fait que la modernité, comme l'affirme Foucault, réside dans la rupture des mots et de la réalité et le quadrillage spontané par les mots de la connaissance des choses ${ }^{2}$.

À la fin de l'année 1964, Claude Simon achève de se positionner dans le champ littéraire. Sa position, fondée sur une proximité à Robbe-Grillet et donc à Barthes, est proche du structuralisme, et de l'anthropologie discursive et symbolique que fonde cette pensée. Ceci conditionne constamment le dialogue avec Sartre, dont on peut dire qu'il est permanent dès La Corde raide. Sartre, il est vrai, nous l'avons constaté, sait occuper le champ littéraire, par ses constants dégagements et ses revirements paradoxaux, par un art de la provocation où l'on doit faire la part à la générosité romantique. Sur le terrain politique et éthique, tous deux parlent d'un universel : mais l'homme de Sartre demeure soumis à l'histoire présente, économique, sociale, politique, culturelle, tandis que celui de Simon ne saurait

1 Voir «Jean-Paul Sartre répond», entretien avec Bernard Pingaud, L'Arc, $\mathrm{n}^{\circ} 30$, numéro spécial « Sartre aujourd'hui », octobre 1966, p. 87-96.

2 Michel Foucault, Les Mots et les choses, Paris, Gallimard, 1966, p. 315. Sur ces points, voir, outre l'entretien publié dans L'Arc, le texte de Sartre « L'anthropologie » (Situations IX, Paris, Gallimard, 1972). 
être l'homme de l'histoire présente. C'est non pas le faire sartrien, mais le dire du structuralisme qui conditionnent la définition de l'écrivain et de l'art, dans leur fonction archéologique soupçonneuse et donc révolutionnaire. En ce sens, Le Palace, qui tourne en dérision la révolution, et donc l'impuissance du faire humain, et qui fait la révolution du langage romanesque, est aussi un récit anti-sartrien. 